\title{
Streptococcus agalactiae em gestantes: prevalência de colonização e avaliação da suscetibilidade aos antimicrobianos
}

\author{
Streptococcus agalactiae in pregnant women: prevalence of colonization and antimicrobial \\ susceptibility evaluation
}

Irina Lermontov Borger ${ }^{1}$, Rachel Elise Cerqueira d'Oliveira ${ }^{2}$, Angela Christina Dias de Castro ${ }^{3}$, Silvia Susana Bona de Mondino ${ }^{4}$

\section{RESUMO}

Objetivos: verificar a ocorrência de colonização por Streptococcus agalactiae em gestantes e avaliar a suscetibilidade das amostras isoladas aos antimicrobianos. Métodos: foram avaliadas 167 grávidas entre a $32^{\mathrm{a}}$ e a $41^{\mathrm{a}}$ semana de gestação, independente da presença ou não de fatores de risco, atendidas no ambulatório de pré-natal entre fevereiro de 2003 e fevereiro de 2004. O material vaginal/anal, colhido com um único $s w a b$, foi inoculado em caldo Todd-Hewitt acrescido de ácido nalidíxico $(15 \mu \mathrm{g} / \mathrm{mL})$ e gentamicina $(8 \mu \mathrm{g} / \mathrm{mL})$, com posterior subcultura no meio de ágar sangue. A identificação foi feita por meio da avaliação da morfologia e tipo de hemólise das colônias no meio de ágar sangue, teste da catalase, teste de cAMP e testes sorológicos. A avaliação da suscetibilidade aos antimicrobianos foi realizada pelos testes de difusão e de diluição em ágar. A análise estatística foi realizada por meio do teste de $\chi^{2}$; valores de $p<0,05$ foram considerados significativos. Resultados: a freqüência de colonização foi de $19,2 \%$, sem diferenças significativas com relação à idade, número de gestações, ocorrência de abortos e presença ou ausência de diabete melito $(\mathrm{p}>0,05)$. Todas as 32 amostras isoladas foram sensíveis a penicilina, cefotaxima, ofloxacina, cloranfenicol, vancomicina e meropenem. A resistência a eritromicina e clindamicina foi detectada em 9,4 e 6,2\% das amostras, respectivamente. Conclusões: a incidência relativamente elevada $(19,2 \%)$ de colonização por $S$. agalactiae entre as gestantes avaliadas e o isolamento de amostras resistentes, especialmente aos antimicrobianos recomendados nos casos de alergia à penicilina, enfatizam a importância de detectar esta colonização no final da gravidez, associada à avaliação da suscetibilidade aos antimicrobianos, para uma prevenção eficaz da infecção neonatal.

PALAVRAS-CHAVE: Estreptococo grupo B; Colonização; Gravidez; Estreptococcus agalactiae; Resistência microbiana a drogas; Prevalência

\section{ABSTRACT}

Purpose: to verify the occurrence of colonization by Streptococcus agalactiae in pregnant women attended at the prenatal outpatient clinic of the Teaching Maternity Hospital of Rio de Janeiro University (UFRJ) and to evaluate the susceptibility of the isolates to antimicrobial agents. Methods: a total of 167 pregnant women between the 32nd and 41st week of gestation, regardless of risk factors, attended at the antenatal clinic between February 2003 and February 2004, were evaluated. The vaginal/anal material, collected by the same swab, was inoculated in Todd-Hewitt broth to which nalidixic acid $(15 \mu \mathrm{g} / \mathrm{mL})$ and gentamicin $(8 \mu \mathrm{g} / \mathrm{mL})$ were added, with following subcultures onto sheep blood-agar. Identification was carried out observing colony morphology and beta-hemolysis type on blood-agar, catalase, cAMP, and serological tests. The antimicrobial susceptibility testing used agar diffusion and agar dilution methods. Statistical analysis was performed by the $\chi^{2}$ test with the level of significance set at $p<0,05$. Results: the frequency of colonization was $19.2 \%$, with no significant differences when age, number of gestations, number of abortions and the presence or absence of diabetes mellitus were compared $(\mathrm{p}>0.05)$. All 32 isolated strains were susceptible to penicillin, cefotaxime, ofloxacin, chloramphenicol, vancomycin and meropenem. Resistance to erythromycin and clindamycin was detected in 9.4 and $6.2 \%$ of the isolates, respectively. Conclusions: the relatively high incidence (19.2\%) of colonization by $S$. agalactiae among the evaluated

Trabalho realizado na Universidade Federal Fluminense - UFF - Rio de Janeiro (RJ) - Brasil.

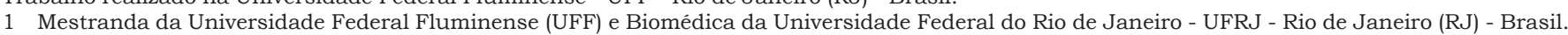

2 Doutoranda do Instituto de Microbiologia Prof. Paulo de Góes da Universidade Federal do Rio de Janeiro - UFRJ - Rio de Janeiro (RJ) - Brasil.

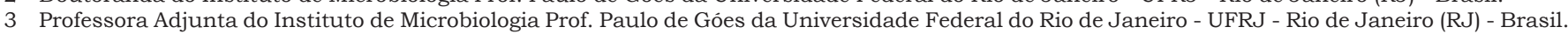

4 Professora Adjunta do Departamento de Patologia da Universidade Federal Fluminense - UFF - Rio de Janeiro (RJ) - Brasil.

Correspondência: Silvia Susana Bona de Mondino

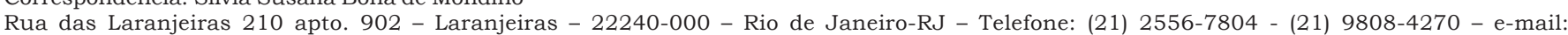
smondino@terra.com.br / smondino@hospital.huap.uff.br 
pregnant women and the recovery of antimicrobial resistant strains, especially those recommended in cases of penicillin allergy, emphasize the importance, for a correct prevention of neonatal infections, of detecting colonization at the end of pregnancy and evaluating antimicrobial susceptibility.

KEYWORDS: Group B streptococcus; Colonization; Pregnancy; Streptococcus agalactiae; Drug resistance, microbial; Prevalence

\section{Introdução}

Streptococcus agalactiae - estreptococo do grupo B (EGB) faz parte da microbiota de membranas mucosas de seres humanos e animais, colonizando principalmente os tratos intestinal e geniturinário. A grande relevância médica deste microrganismo está na contaminação de neonatos, ocasionando quadros graves de septicemia, pneumonia e meningite ${ }^{1}$.

Na década de 80 foi verificada uma importante diminuição nas taxas de sepse neonatal com o uso de quimioprofilaxia em gestantes colonizadas pelo EGB. Em 1996, o Centers for Disease Control (CDC) elaborou guia para a prevenção da infecção precoce do neonato, recomendando a prescrição de quimioprofilaxia em duas situações: 1) em todas as grávidas colonizadas com EGB, de acordo com os resultados da cultura realizadas entre a $35^{\mathrm{a}}$ e a $37^{\mathrm{a}}$ semana de gravidez, ou 2) nas grávidas que, não tendo sido submetidas à pesquisa de colonização pelo EGB, apresentarem algum dos fatores de risco para a contaminação da criança, como tempo de ruptura de membrana maior ou igual a 18 horas, temperatura igual ou superior a $38^{\circ} \mathrm{C}$ durante o parto e/ou prematuridade. Posterior atualização desse guia, realizada em 2002, enfatiza a maior eficácia do protocolo baseado na pesquisa de colonização pelo EGB quando comparado ao protocolo baseado nos fatores de risco. Estas recomendações foram adotadas pelo American College of Obstetricians and Gynecologists (ACOG) e pela American Academy of Pediatrics. Indica-se a quimioprofilaxia, também, nas gestantes que apresentaram bacteriúria por EGB durante a gravidez ou que tiveram um filho com doença precoce por $\mathrm{EGB}^{1,2}$

Alguns autores discordam da utilização do protocolo baseado na cultura, por ser dispendioso e não ter evidenciado maior efetividade do que o fundamentado, apenas, nos fatores de risco ${ }^{3}$. Entretanto, a maioria dos trabalhos mostra que a estratégia baseada na pesquisa de colonização por EGB para definir o uso da quimioprofilaxia antibiótica intraparto é mais eficaz, principalmente levando em conta que grande parte das gestantes colonizadas não possuem nenhum dos fatores de risco para infecção do neonato ${ }^{1,4-6}$.
$\mathrm{O}$ indice de isolamento do microrganismo depende de vários fatores relacionados com a coleta e a metodologia laboratorial empregada. Assim, tem sido demonstrado que a coleta de espécime vaginal/anal, executada com o mesmo swab, apresenta um índice de positividade duas vezes superior ao da coleta de material apenas vaginal ${ }^{7}$. Por outro lado, em espécimes com baixa densidade bacteriana, há comprometimento da viabilidade celular quando os swabs, mesmo preservados em meio de transporte, ficam estocados em temperatura igual ou superior a $30^{\circ} \mathrm{C}$ ou durante mais de quatro $\operatorname{dias}^{8}$. Quanto à metodologia laboratorial, a utilização de meio seletivo, contendo agentes antimicrobianos para inibir o crescimento de outros microrganismos, aumenta a possibilidade de crescimento do EGB em aproximadamente $50 \%{ }^{1}$.

Os antibióticos habitualmente utilizados na quimioprofilaxia são penicilina ou ampicilina e, nos casos de alergia, eritromicina ou clindamicina. Vários estudos têm evidenciado o aparecimento, em diferentes países, de cepas de EGB resistentes a estes últimos antimicrobianos, o que os torna inadequados como opção de escolha tanto para a quimioprofilaxia como para o tratamento de infecções ${ }^{9-11}$.

Desde que os estudos visando avaliar a incidência de colonização por EGB em gestantes são raros em nosso meio, este trabalho teve como objetivo determinar a taxa de colonização em gestantes e avaliar a suscetibilidade aos antimicrobianos das amostras isoladas.

\section{Métodos}

O trabalho foi estudo de prevalência prospectivo com a coleta de material vaginal/anal em 167 gestantes atendidas no ambulatório de pré-natal, no periodo de fevereiro de 2003 a fevereiro de 2004. O projeto foi aprovado pela Comissão de Ética em Pesquisa. Todas as pacientes assinaram termo de consentimento para a coleta de seu espécime clínico e os resultados foram notificados ao corpo médico.

A população avaliada incluiu grávidas entre a $32^{\text {a }}$ e a $41^{\text {a }}$ semana de gestação, 59 primíparas e 
108 multíparas, sem sinais clínicos de infecção no momento da coleta; em 50 mulheres foi verificada a ocorrência de abortos anteriores; 14 eram diabéticas e 153 não diabéticas. A faixa etária variou entre 14 e 41 anos.

A coleta e o processamento do material foram realizados de acordo com as recomendações do $\mathrm{CDC}^{1}$. A coleta de material vaginal/anal foi feita pelos obstetras, utilizando um único swab, com posterior colocação do mesmo em meio de transporte e armazenado em temperatura ambiente. $\mathrm{O}$ tempo transcorrido entre a coleta e o processamento do material foi, no máximo, de quatro dias. No laboratório, o material foi inoculado em caldo Todd-Hewitt (Difco Laboratories, Detroit, MI, EUA) adicionado de gentamicina $(8 \mu \mathrm{g} / \mathrm{mL}$ ) (Sigma) e ácido nalidíxico $(15 \mu \mathrm{g} / \mathrm{mL}$ ) (Acros Organics, NJ, EUA). Após incubação de 6 horas a $35^{\circ} \mathrm{C}$, foi realizada subcultura no meio de ágar sangue (tryptic soy agar, Difco, acrescido de $5 \%$ de sangue desfibrinado de carneiro).

As colônias sugestivas de EGB, acinzentadas, circundadas por halo discreto de hemólise total ( $\beta$-hemólise) ou não hemolíticas, foram submetidas à coloração de Gram e observadas ao microscópio. Os cocos gram-positivos foram testados quanto à capacidade de produzir catalase e o fator cAMP. Os microrganismos catalase-negativos e cAMP positivos foram submetidos a sorogrupagem ${ }^{12}$ utilizando o teste comercial para identificação de estreptococos (Streptoccocal Grouping Kit, Oxoid), de acordo com as recomendações do fabricante.

A avaliação da suscetibilidade das amostras aos antimicrobianos foi realizada pela utilização do teste de difusão em ágar, conforme as recomendações do National Committee for Clinical Laboratory Standards (NCCLS) ${ }^{13}$. Os antibióticos testados foram: penicilina (10 U); cefotaxima (30 $\mu \mathrm{g})$; eritromicina $(15 \mu \mathrm{g})$; clindamicina $(2 \mu \mathrm{g})$; ofloxacina (5 $\mu \mathrm{g})$; cloranfenicol (30 $\mu \mathrm{g})$; vancomicina (30 $\mu \mathrm{g})$, todos obtidos da CECON (São Paulo, SP, Brasil), e o meropenem (10 $\mu \mathrm{g})$, obtido da Oxoid (Basingstoke, Hampshire, England).

Foram realizadas as determinações da concentração mínima inibitória de eritromicina, clindamicina e penicilina em todas as amostras, por meio do método de diluição em ágar, de acordo com as recomendações do NCCLS ${ }^{14}$

A análise estatística foi realizada pelos testes de $\chi^{2}$ utilizando o programa Statistical Package for the Social Sciences versão 10.0, para comparar as freqüências de colonização em relação a idade, número de gestações, presença ou ausência de diabete melito e número de abortos. Os valores de $\mathrm{p}<0,05$ foram considerados significativos.

\section{Resultados}

A freqüência de colonização vaginal/anal por EGB detectada foi de 19,2\% (IC 95\%). A comparação da ocorrência de colonização por S. agalactiae de acordo com a idade, número de gestações anteriores e de abortos não mostrou diferenças estatisticamente significativas $(\mathrm{p}>0,05)$ (Tabela 1$)$. Das 153 gestantes não diabéticas estudadas, 29 (19\%) estavam colonizadas, ao passo que das 14 diabéticas, $3(21,4 \%)$ estavam colonizadas. A comparação da freqüência de colonização por $S$. agalactiae entre os dois grupos (diabéticas e não diabéticas) não mostrou significância estatística entre esses fatores $(p>0,05)$.

Tabela 1 - Prevalência de colonização vaginal/anal por estreptococo do grupo B em gestantes segundo faixa etária e antecedentes obstétricos.

\begin{tabular}{lc}
\hline Dados das gestantes & $\begin{array}{c}\mathbf{N}^{0} \text { de colonizadas/ } \\
\mathbf{N}^{0} \text { de gestantes avaliadas (\%) }\end{array}$ \\
\hline Faixa etária & $3 / 9(33,3)$ \\
$15-19$ & $9 / 44(20,5)$ \\
$20-24$ & $10 / 50(20,0)$ \\
$25-29$ & $8 / 37(21,6)$ \\
$30-34$ & $2 / 27(7,4)$ \\
35 e acima & \\
$\mathrm{N}^{0}$ de gestações & $15 / 59(25,4)$ \\
1 & $7 / 49(14,3)$ \\
2 & $7 / 32(21,8)$ \\
3 & $2 / 22(9,1)$ \\
4 & $1 / 5(20,0)$ \\
5 ou mais & \\
$\mathrm{N}^{0}$ de abortos & \\
0 & $27 / 115(23,5)$ \\
2 & $4 / 39(10,3)$ \\
\hline ou mais & $0,05)$. \\
\end{tabular}

Todas as amostras de EGB foram sensiveis a penicilina, cefotaxima, ofloxacina, cloranfenicol, meropenem e vancomicina. A resistência a eritromicina foi verificada em três amostras (3/ $32 ; 9,4 \%)$, duas das quais foram, concomitantemente, resistentes a clindamicina $(6,2 \%)$. As amostras resistentes foram isoladas de gestantes que não apresentaram nenhuma característica peculiar em relação às outras grávidas das quais foram isoladas amostras sensiveis a todos os antimicrobianos testados. 


\section{Discussão}

A freqüência de colonização por EGB durante a gravidez é variável, estando relacionada com diferenças socioculturais, geográficas e com as metodologias bacteriológicas empregadas, entre outras. Assim, em estudos realizados na Turquia, a taxa de colonização detectada foi de $8 \%^{15}$, nos Estados Unidos entre 18,6 e $21,1 \%{ }^{16}$ e no Chile, de $19,9 \%{ }^{17}$.

Os dados brasileiros são relativamente escassos, com indices de colonização inferiores ao valor de 19,2\% verificado neste trabalho. Em estudo realizado em São Paulo, as taxas detectadas foram baixas, de 1,9\%, decorrente, talvez, da coleta de espécime apenas vaginal e da inoculação em meio de cultura não seletivo ${ }^{18}$. Entretanto, numa avaliação realizada em Salvador, onde foi utilizada a mesma metodologia de coleta e laboratorial empregada neste estudo, o índice foi de $6,9 \%$, inferior ao detectado em nosso trabalho ${ }^{19}$. Estes resultados mostram a presença de vários fatores influenciando nos índices de colonização pelo EGB nas gestantes, além dos meramente metodológicos, particularmente o perfil da população estudada.

Para implementar adequada quimioprofilaxia, e assim diminuir a incidência de sepse neonatal pelo EGB, é fundamental que o laboratório de bacteriologia informe rapidamente a presença do microrganismo e sua sensibilidade aos antimicrobianos. Neste estudo os resultados foram relativamente rápidos, não ultrapassando, na maioria das vezes, os três dias para a identificação da bactéria e cinco dias para o teste de suscetibilidade.

Desde que não foram verificadas diferenças significativas nas taxas de colonização por EGB quando comparadas variáveis como faixa etária, número de gestações e de abortos, estes fatores não devem ser utilizados como critério para prescrever a quimioprofilaxia. Entretanto, deve-se ressaltar que quase a metade do total das grávidas colonizadas $(15 / 32 ; 46,9 \%)$ eram primiparas. Neste grupo, os fatores de risco relativos a gestações anteriores, como história prévia de sepse neonatal e prematuridade, são inexistentes, o que torna mais relevante a utilização do protocolo baseado na cultura para proceder à quimioprofilaxia. Em estudo realizado em hospital privado em São Pau10 , foi verificado que $60 \%$ dos neonatos com infecção neonatal invasiva nasceram de mães primíparas $^{20}$

Alguns autores verificaram maior freqüência de colonização por S. agalactiae em gestantes diabéticas quando comparadas com as não diabéticas ${ }^{21}$; outros, entretanto, não comprovaram esta associação ${ }^{22}$. No presente estudo não foram verificadas diferenças significativas ao comparar colonização por EGB entre as gestantes diabéticas e as não diabéticas. Entretanto, estes resultados não podem ser considerados como definitivos, em decorrência do reduzido número de grávidas diabéticas avaliadas (14/167; 8,4\%).

Desde a implantação dos protocolos de prevenção das doenças do neonato, o uso de eritromicina e clindamicina tem aumentado, sobretudo em pacientes alérgicas a penicilina. Ao mesmo tempo, houve aumento nas taxas de resistência do EGB a esses antimicrobianos, de acordo com o verificado em estudos realizados em diferentes países. Assim, em relação a eritromicina, os maiores indices de resistência foram detectados nos Estados Unidos da América $(30 \%)^{23}$, na França $(21,4 \%)^{24}$, na Turquia $(20 \%)^{15} \mathrm{e}$, em menor proporção, em Portugal $(10,7 \%)^{25}$. Em relação à clindamicina, as taxas de resistência, de modo geral, são inferiores, com índices de $17,5 \%$ na França $^{24}, 11,4 \%$ nos Estados Unidos da América ${ }^{23}$ e $9,9 \%$ em Portugal ${ }^{25}$.

No Brasil, a detecção de amostras de $S$. agalactiae resistentes a esses antimicrobianos vem demonstrando tendência de aumento. Neste estudo, as taxas de resistência a eritromicina $(9,4 \%)$ e clindamicina $(6,2 \%)$ foram superiores às de 5,4 e $1,1 \%$, respectivamente, detectadas em amostras clínicas de $S$. agalactiae isoladas de pacientes infectados no período de 1994 a 1999, no Rio de Janeiro ${ }^{11}$.

A freqüência relativamente elevada de colonização por EGB verificada neste estudo $(19,2 \%)$, assim como a detecção de amostras resistentes aos antibióticos recomendados pelo CDC nos casos de alergia a penicilina, devem alertar a classe médica acerca da importância de incluir, no exame pré-natal, a realização de cultura para pesquisa da colonização por EGB com a avaliação sistemática da suscetibilidade aos antimicrobianos, a fim de proceder a uma escolha racional do antimicrobiano a ser utilizado na quimioprofilaxia.

Apoio financeiro do Conselho Nacional de Desenvolvimento Científico e Tecnológico (CNPq), Coordenação de Aperfeiçoamento de Pessoal de Nivel Superior (CAPES), Financiadora de Estudos e Projetos (FINEP), Fundação de Amparo à Pesquisa do Estado do Rio de Janeiro (FAPERJ) e Ministério da Ciência e Tecnologia (MCT/PRONEX), Pro-Reitoria de Pesquisa e Pós-graduação da Universidade Federal Fluminense (PROPP/UFF) e Instituto de Microbiologia Prof. Paulo de Góes da Universidade Federal do Rio de Janeiro (UFRJ). 


\section{Referências}

1. Schrag S, Gorwitz R, Fultz-Bultz K, Schuchat A. Prevention of perinatal group B streptococcal disease: revised guidelines from CDC. MMWR Recomm Rep. 2002;51(RR-11):1-22.

2. Apgar BS, Greenberg G, Yen G. Prevention of group $B$ streptococcal disease in the newborn. Am Fam Physician. 2005;71(5):903-10.

3. Yucesoy G, Caliskan E, Karadenizli A, Corakci A, Yucesoy I, Huseyinoglu N, et al. Maternal colonisation with group B streptococcus and effectiveness of a culture-based protocol to prevent early-onset neonatal sepsis. Int $\mathrm{J}$ Clin Pract. 2004;58(8):735-9.

4. Main EK, Slagle T. Prevention of early-onset invasive neonatal group B streptococcal disease in a private hospital setting: the superiority of culture-based protocols. Am J Obstet Gynecol. 2000;182(6):1344-54.

5. De Cueto M, de la Rosa M. Prevención de la infección neonatal por Streptococcus agalactiae. Un tema consolidado. Enferm Infecc Microbiol Clin. 2003;21(4):171- 3 .

6. Schrag SJ, Zell ER, Lynfield R, Roome A, Arnold $\mathrm{KE}$, Craig AS, et al. A population-based comparison of strategies to prevent early-onset group B streptococcal disease in neonates. N Engl J Med. 2002;347(4):233-9.

7. Quinlan JD, Hill DA, Maxwell BD, Boone S, Hoover $\mathrm{F}$, Lense JJ. The necessity of both anorectal and vaginal cultures for group B streptococcus screening during pregnancy. J Fam Pract. 2000;49(5):447-8.

8. Stoner KA, Rabe LK, Hillier SL. Effect of transport time, temperature and concentration on the survival of group B streptococci in amies transport medium. J Clin Microbiol. 2004;42(11):5385-7.

9. De Azavedo JC, McGavin M, Duncan C, Low DE, McGeer A. Prevalence and mechanisms of macrolide resistance in invasive and noninvasive group $B$ streptococcus isolates from Ontario, Canada. Antimicrob Agents Chemother. 2001;45(12):3504-8.

10. Hsueh PR, Teng LJ, Lee LN, Ho SW, Yang PC, Luh KT. High incidence of erythromycin resistance among clinical isolates of Streptococcus agalactiae in Taiwan. Antimicrob Agents Chemother. 2001;45(11):3205-8.

11. D`Oliveira RE, Barros RR, Mendonça CR, Teixeira LM, Castro AC. Susceptibility to antimicrobials and mechanisms of erythromycin resistance in clinical isolates of Streptococcus agalactiae from Rio de Janeiro, Brazil. J Med Microbiol. 2003;52(Pt 11):1029-30.

12. Ruoff KL, Whiley RA, Beighton D. Streptococcus. In: Murray PR, Baron EJ, Pfaller MA, Jorgensen JH, Yolken RH, editors. Manual of clinical microbiology. 8th ed. Washington, DC: American Society for Microbiology; 2003. p. 405-21.

13. National Committee for Clinical Laboratory Standards. Performance standards for antimicrobial disk susceptibility tests for bacteria that grow aerobically. 8th ed. Wayne: NCCLS; 2003. (Approved Standard. NCCLS document M2-A8).

14. National Committee for Clinical Laboratory Standards. MIC testing: supplemental tables. Wayne: NCCLS; 2003. (NCCLS document M100-S13 (M7)).

15. Barbaros I, Murat C, Mehmet V, Ismet TA, Can K, Sukufe D, et al. The colonization incidence of group B streptococcus in pregnant women and their newborns in Istanbul. Pediatr Int. 2005;47(1):64-6.

16. Regan JA, Klebanoff MA, Nugent RP, Eschenbach DA, Blackwelder WC, Lou Y, et al. Colonization with group $\mathrm{B}$ streptococci in pregnancy and adverse outcome. Am J Obstet Gynecol. 1996;174(4):1354-60.

17. Belmar JC, Abarzúa CF, Beker VJ, Gusmán AM, García CP, Oyarzún Ebensperger E. Estudio de sensibilidad antimicrobiana de 183 cepas de Streptococcus agalactiae aisladas en región vaginoperineal de embarazadas en el tercer trimeste. Rev Chil Obstet Ginecol. 2002;67(2):106-9.

18. Carvalho MHB, Bittar RE, Maganha PPAS, Fonseca EVB, Zugaib M. Incidência de colonização vaginal por Streptococcus agalactiae na população geral de gestantes. Rev Bras Ginecol Obstet. 2001;12(3):108-11.

19. Pellegrini R. Freqüência de colonização por Streptococccus agalactiae em gestantes da cidade de Salvador, Bahia. Rev Soc Bras Med Trop. 1999;32(4):451-2.

20. Vaciloto E, Richtmann R, Costa HPF, Kusano EJU, Almeida MFB, Amaro ER. A survey of the incidence of neonatal sepsis by group B streptococcus during a decade in a Brazilian maternity hospital. Braz J Infect Dis.2002;6(2):55-62.

21. Ramos E, Gaudier FL, Hearing LR, Del Valle GO, Jenkins S, Briones D. Group B streptococcus colonization in pregnant diabetic women. Obstet Gynecol. 1997;89(2):257-60.

22. Hammoud MS, Thalib L, Maiyegun SO. The epidemiology of group B streptococcal colonization among obstetrical and newborn populations in Kuwait. Int J Gynaecol Obstet. 2002;76(3):315-6.

23. Biedenbach DJ, Stephen JM, Jones RN. Antimicrobial susceptibility profile among beta-haemolytic Streptococcus spp. collected in the SENTRY Antimicrobial Surveillance Program-North America, 2001. Diag Microbiol Infect Dis. 2003;46(4):291-4.

24. De Mouy D, Cavallo JD, Leclercq R, Fabre R; AFICORPI-BIO Network. Antibiotic susceptibility and mechanisms of erythromycin resistance in clinical isolates of Streptococcus agalactiae: French multicenter study. Antimicrob Agents Chemother. 2001;45(8):2400-2.

25. Figueira-Coelho J, Ramirez M, Salgado MJ, MeloCristino J. Streptococcus agalactiae in a large Portuguese teaching hospital: antimicrobial susceptibility, serotype distribution and clonal analysis of macrolide-resistant isolates. Microb Drug Res. 2004;10(1):31-6. 